\title{
JAK ACADEMY in Rheumatoid Arthritis (RA)
}

\section{Edited by the Scientific Committee}

\section{Roberto Caporali ${ }^{1}$, Marcello Govoni ${ }^{2}$, Giovanni Lapadula ${ }^{3}$, Luigi Sinigaglia ${ }^{4}$, Guido Valesini ${ }^{5}$}

1 Associate Professor of Rheumatology, Pavia University, Director of the Early Arthritis Clinic of the IRCCS [Istituto di Ricovero e Cura a Carattere Scientifico (Scientific Institute for Research, Hospitalisation and Healthcare)] Foundation Policlinico San Matteo di Pavia ['San Matteo' General Hospital of Pavia]

2 Associate Professor of Rheumatology, University of Ferrara. Head UOC Reumatologia - Azienda Ospedaliero Universitaria S. Anna - Ferrara (Ioc. Cona)

\section{Full Professor, Dipartimento dell'Emergenza e dei Trapianti di organi (DETO) [Department of Emergency} and Organ Transplants]

\section{Director of the Department of Rheumatology and Medical Sciences Centro Ortopedico Traumatologico} Gaetano Pini-CTO, Milano

5 Full Professor of Rheumatology, Director of the Rheumatology Specialisation School, Sapienza Università di Roma ['Sapienza' University of Rome]. Coordinator of the Research Doctorate in Clinical and Experimental Rheumatology

\section{Abstract}

Rheumatoid arthritis (RA) is a debilitating autoimmune disease characterized by chronic inflammation with subsequent joints destruction, increasing disability and mortality. Inflammatory joint pain is usually the first symptoms, frequently associated with nonspecific systemic symptoms that may include fatigue. The principal target of chronic inflammation in RA is represented by the synovial membrane.

In RA the inflammatory milieu in the synovial compartment is regulated by a complex cytokine

\section{Correspondence to:}

Roberto Caporali

e-mail: caporali@smatteo.pv.it

Accepted: 5/4/2018 - Published online: 11/4/2018

(C) 2018 The Authors. This article is published by HPS Srl and licensed under Creative Commons Attribution-NC-ND 4.0 International (CC BY-NC-ND 4.0). Any commercial use is not permitted and is subject to Publisher's permissions. Full information is available at www.aboutpharma.com/publishing/riviste/aboutopen/ and chemokine network, leading to the induction and aggravation of the inflammatory response by activating endothelial cells and attracting immune cells to the synovial compartment. Activated fibroblasts, together with activated $\mathrm{T}$ cells and $\mathrm{B}$ cells, monocytes and macrophages, ultimately trigger osteoclast generation with bony erosions.

Cytokine are soluble messengers used by immune cells to communicate; in RA an imbalance between pro-inflammatory and anti-inflammatory cytokines has been well demonstrated, leading

to maintain and aggravate inflammation. Over the past 20 years, our knowledge of the intracellular pathways downstream of cytokine receptors has greatly increased, with particular interest in the Janus kinase (JAK)-STAT pathway; the inhibition of such intracellular enzymes represents a novel way to simultaneously inhibit multiple cytokines.

The management of RA has changed radically over the last 20 years. Nowadays, due to the availability of a larger number of effective treatment options and 
more stringent classification criteria for the disease, it is possible to control the symptoms of RA by aiming to achieve long-term clinical outcomes such as remission or at least low disease activity (treat to target approach).

The latest update of the EULAR recommendations for the treatment of RA maintain CSDMARD (conventional systemic DMARDs) as the first line treatment, with methotrexate (MTX) still identified as the anchor drug, to which it is possible to add, in failure patients with negative prognostic factors, a biological drug or a tsDMARD (target synthetic DMARD), i.e JAK inhibitors.

In November 2012, the Food and Drug Administration (FDA) approved the use of tofacitinib $5 \mathrm{mg} /$ bid orally for the treatment of RA, which was approved by the European Medicines Agency (EMA), in March 2017, in combination with methotrexate (MTX) for the treatment of moderate to severe active RA in adult patients who have responded inadequately to, or who are intolerant to one or more DMARDs. It can be given as monotherapy in case of intolerance to MTX or when treatment with MTX is inappropriate. In February 2017, EMA also approved the use of baricitinib 2 and $4 \mathrm{mg}$ for the same indication.

We believe that a deeper knowledge on this new class of DMARDs is needed and "JAK Academy RA" can represent a valid tool to engage in more and more conscious evaluations of the most suitable treatment strategy for each patient. The proposed educational process consists of 15 video lectures which, starting from a description of the pathogenesis of RA, facilitates in-depth learning about the mechanisms involved in intracellular signalling, and the JAK pathway in particular, and the consequences of its inhibition, up to and including the clinical data on the JAK inhibitors approved for the treatment of RA. We report the program below for the different video lectures.

RA: a new treatment approach

- "Immune and inflammatory responses: the driving forces in the pathogenesis of RA" - C. Alessandri

- "Cytokines, biological process and RA and signal through different pathways" - F. Ciccia

- "From extracellular to intracellular: signal transduction, JAK pathway story and description and the biological significance of signaling through different JAK combinations" - F. Conti

- "Pharmacological aspects of JAK inhibitors" - C. Blandizzi

- "Who may need new treatment options for RA? (unmet need in RA)"- F. lannone

JAKis efficacy

- "JAK inhibitors: clinical efficacy in a broad patients population" - E. G. Favalli

- "JAK inhibitors: a new chance for RA patients intollerant to MTX" - A. Doria

- "JAK inhibitors: the radiographic progression" - M. A. Cimmino

- "JAK inhibitors: from patients perspective" - F. Salaffi

JAKis safety

- "JAK inhibitors: infections risk" - L. Sinigaglia

- "JAK inhibitors: CV risk" - F. Atzeni

- "JAK inhibitors: risk/benefit balance from RW experience" - R. Caporali

- "JAK inhibitors: screening and monitoring" - C. Girmenia

JAKis positioning

- "New therapeutic algorithms" - M. Govoni

www.pfizerpro.it/jak-academy-lectures-ondemand-ra-disclaimer

\section{Disclosures}

JAK Academy RA is supported by Pfizer as an educational grant.

Conflict of interest: The authors declare they have no conflict of interest related to this article. 